\title{
Learning constraints in spreadsheets and tabular data
}

\author{
Samuel Kolb ${ }^{1} \cdot$ Sergey Paramonov $^{1}$ (D) Tias Guns ${ }^{2}$. \\ Luc De Raedt ${ }^{1}$
}

Received: 25 September 2016 / Accepted: 28 April 2017 / Published online: 5 June 2017

(C) The Author(s) 2017

\begin{abstract}
Spreadsheets, comma separated value files and other tabular data representations are in wide use today. However, writing, maintaining and identifying good formulas for tabular data and spreadsheets can be time-consuming and error-prone. We investigate the automatic learning of constraints (formulas and relations) in raw tabular data in an unsupervised way. We represent common spreadsheet formulas and relations through predicates and expressions whose arguments must satisfy the inherent properties of the constraint. The challenge is to automatically infer the set of constraints present in the data, without labeled examples or user feedback. We propose a two-stage generate and test method where the first stage uses constraint solving techniques to efficiently reduce the number of candidates, based on the predicate signatures. Our approach takes inspiration from inductive logic programming, constraint learning and constraint satisfaction. We show that we are able to accurately discover constraints in spreadsheets from various sources.
\end{abstract}

Keywords Constraint learning · Tabular constraint learning · Spreadsheets · Excel · Constraint programming $\cdot$ Constraint discovery $\cdot$ Machine learning

\section{Introduction}

Millions of people across the world use spreadsheets every day. The tabular representation of the data is often intuitive, and the programming of functions in individual cells is quickly learned. However, large and complex sheets, possibly with multiple tables and relations

Editors: Kurt Driessens, Dragi Kocev, Marko Robnik-Šikonja, and Myra Spiliopoulou.

Samuel Kolb and Sergey Paramonov: Shared first author.

Sergey Paramonov

sergey.paramonov@cs.kuleuven.be

1 KU Leuven, Leuven, Belgium

2 Vrije Universiteit Brussel, Brussels, Belgium 
between them, can be hard to handle. Many end-users lack the understanding of the underlying structures and relations in such sheets and the data they contain. This is especially the case when spreadsheets have been exported from other software such as Enterprise Resource Planning (ERP) systems. In this case, often a Comma Separated Values (CSV) format is used meaning that all formulas are lost, including inter-sheet formulas and relations. Even in manually created spreadsheets, it can be challenging to be consistent and correct with formulas across all tables. For example, the influential Reinhart-Rogoff economical paper "Growth in a Time of Debt" (Reinhart and Rogoff 2010) had some of its claims contested (Herndon et al. 2013) after an investigation showed that the used Excel sheets contained mistakes in formulas.

Such issues and mistakes could be overcome through the development of intelligent userassisting tools that would be able to automatically discover what constraints and formulas hold in a spreadsheet. This is the problem we study in this paper. We envision that automatic constraint discovery can enable: auto-completion, error checking, formula suggestion, rich import and data compression. Auto-completion can help users quickly populate spreadsheets by suggesting values for unfilled cells based on constraints that have previously been detected within the spreadsheet. Error checking can identify erroneous values in spreadsheets, for example, while typing, by noticing that previously discovered constraint are violated by newly entered values, or offline by selectively leaving out values. Both of these applications can facilitate the use of spreadsheets even for users who do not explicitly use formulas at all. Furthermore, these applications are able to explain what constraints are responsible for suggesting or rejecting a value. Other potential applications include formula suggestion, which is a variant of auto-completion that does not suggest a value but provides a formula which computes the user provided value. This setting can help users in finding the formula to perform a certain task or finding the right syntax for a formula. Rich import denotes importing semi-structured data from another source such as CSV files and extracting the formula's that were used implicitly or explicitly. A similar approach could be used to compress the data: instead of storing all data in a file, one can identify rows or columns generated by formula and store just that formula instead of the values.

To this aim, we investigate whether machine learning and knowledge discovery techniques can be used to learn constraints (formulas and other relations) in spreadsheet data in an unsupervised way. From a machine learning point of view this is an unconventional problem because the data is in tabular form, but the constraints we wish to learn can involve both rows and columns of the table. Being unsupervised, there is no labeled information either, although for every possible function one can verify whether a certain input satisfies the definition. From a data mining point of view, the data is relational on the one hand, since one can have multiple tables with relationships between them, but also consists of mixed textual and numeric types. More closely related is clausal discovery (De Raedt and Dehaspe 1997; Lallouet et al. 2010), learning CSP constraints (Bessiere et al. 2013; Bessière et al. 2005; Beldiceanu and Simonis 2012) and dependency discovery in databases (Savnik and Flach 2000). But an important difference remains that we want to learn constraints on both columns and rows over integer, floating point as well as textual data. Our inspiration comes from work on program synthesis, in particular Flashfill (Gulwani 2011), where the definition of a string-manipulation function is learned in spreadsheet data from very few examples.

The question that we ask in this paper is: is it possible to learn the set of constraints present in a spreadsheet, from just tabular spreadsheet data? The main challenge is the number of possible constraints and combinations of rows and columns that need to be considered as input to the constraints. To answer this challenge we propose a general-purpose method and system, named TaCLe (from: Tabular Constraint Learner, pronounced "tackle"), for discovering rowwise and column-wise constraints. Constraints on contiguous rows and columns are most 
common in spreadsheets, with semantically related items stored in rows or columns and formula's usually ranging over contiguous ranges, as formulas parallel to a table are often dragged over its entire span. Our contributions are as follows:

- we define the tabular constraint learning problem, where the goal is to find constraints that range over entire rows or columns in an unsupervised way;

- we propose an effective two-stage generate-and-test method where the first stage reasons only over properties of contiguous blocks of rows/columns, and the second stage continues to investigate individual rows and columns and their content;

- furthermore, in the first stage we use a constraint solver to efficiently enumerate all combinations of maximally contiguous blocks compatible with the arguments of the candidate constraints

- experiments on different publicly available spreadsheets show that the system is able to extract constraints with high precision and recall.

This paper is organized as follows. Section 2 discusses related work. Section 3 introduces the relevant concepts and defines the problem formally while Sect. 4 presents our approach. This is evaluated in Sect. 5 after which we show how to realize two of the motivating applications using our system in Sect. 6, and we conclude in Sect. 7.

\section{Related work}

TaCLe combines ideas from several existing approaches and different fields of research.

First, it borrows techniques from logical and relational learning (De Raedt 2008), as it discovers constraints in multiple relations or tables. Unlike typical logical and relational learning approaches, it focuses on data in spreadsheets and constraints involving both columns and rows in the sheets. Furthermore, it derives a set of simple "atomic" constraints rather than a set of rules that each consist of a conjunction of literals as in clausal discovery (De Raedt and Dehaspe 1997; Lallouet et al. 2010). Nevertheless, several ideas from logical and relational learning proved to be useful, such as the use of a canonical form, a refinement graph, and pruning for redundancy. Tabular constraint learning also connects with the logical generalization of given formulas in a spreadsheet (Isakowitz et al. Jan 1995), where the existing formulas are abstracted by introducing parameters in the place of particular cell references. The key difference here is that we discover constraints/formulas in the data, we do not have or use any prior information on the formulas in a spreadsheet.

Second, there exist a number of algorithms in the constraint programming community that induce constraints from one or more examples and questions. Two well-known approaches include ModelSeeker (Beldiceanu and Simonis 2012) and Quacq (Bessiere et al. 2013). The former starts from a single example in the form of a vector of values and then exhaustively looks for the most specific constraints that hold given a large constraint library. To this aim, it cleverly enumerates different de-vectorized tables; for instance, if the initial vector was of dimension $1 \times 20$, it would consider rearrangements of size $2 \times 10,4 \times 5,5 \times 4, \ldots$ and Modelseeker would then look for both column, row and even diagonal constraints. Key differences with our work are that we use spreadsheets with multiple tables and that ModelSeeker's constraints are restricted to integer values and constraints that are typical for the constraint programming community, e.g., aimed at combinatorial optimization and scheduling. Conacq [3] acquires a conjunction of constraints using techniques inspired by Mitchell's version space algorithm to process examples; its successor Quacq uses active learning to query the 
user. Each of the examples/queries is processed for each constraint, hence an important issue is how fast the algorithm converges to the entire solution set (e.g. for Sudoku). The focus is again on integer variables and typical combinatorial optimisation constraints. In a similar spirit the field of equation discovery (Todorovski 2010) focuses, guided by heuristics based on the structure of physical equations and unit measures, on the discovery of physical formulas over numbers and times series. However, they focus specifically on noisy physical data and formulas, e.g., a single variable can take values from a series of measurements to get a regression parameter estimate or they apply specific data transformation such as numerical calculation of time and partial derivatives. In other words, the method works with approximations and the equation structures are heavily biased towards parameter estimation, while our goal is to discover hard constraints that hold over a subset of the rows and columns.

Third, our work borrows from the seminal work of Gulwani (2011) on program synthesis for string manipulation in tabular data, which is incorporated in Microsoft's Excel. In FlashFill, the end-user might work on a table containing the surnames (column A) and names (column B) of scientists. The first row might contain the values A1 = "Curie" and B1 = "Marie", and the user might then enter in cell C1 the value "M.C.". At that point FlashFill generates a program that produces the output "M.C." for the inputs "Curie" and "Marie", and also applies it to the other scientists (rows) in the table. While it might need an extra example to deal with names such as "Bell", "Alexander Graham" and determine whether it should output "A.B." or "A.G.B.", FlashFill learns correct programs from very few examples. Flashfill has been extended to, e.g., FlashExtract (Le and Gulwani 2014) to produce a set of tables starting from an unstructured document (e.g., a txt or HTML file) with a set of examples marked for extraction. However, unlike our approach, Flashfill requires the user to identify explicitly the desired inputs/outputs from the function as well as to provide explicitly some positive and sometimes also negative examples. In contrast, our approach is unsupervised, although it would be interesting to study whether it could be improved through user interaction and through user examples. Furthermore, FlashFill handles only single tables with textual data to obtain a string transformation program, while the examples we consider contain multiple tables with mixed numeric and textual data and multiple constraints in them. An interesting open issue for all of these techniques, with the exception of logical and relational learning, is how to deal with possibly incomplete or noisy data.

Fourth, also related to this line of research is the work on deriving constraints in databases such as functional and multi-valued dependencies (Savnik and Flach 2000; Mannila and Räihä 1994) although that line of research has focused on more specialized techniques for specific types of constraints. Many of the discovery techniques rely on the database schema (Flach and Savnik 1999), which is not available in our case. Techniques that work directly on the data have been investigated for the discovery of functional dependencies between attributes (Huhtala et al. 1999). The constraints and formulas we wish to learn go beyond that, including arithmetic, conditional arithmetic and fuzzy lookups, for example.

Finally, worth mentioning is BayesDB (Mansinghka et al. 2015) and Tabular (Gordon et al. 2013), two recent probabilistic modeling languages that have been specifically designed for dealing with relational data in tabular form and that can, as FlashFill, also automatically fill out missing values in a table. However, unlike the other mentioned approaches, it is based on an underlying probabilistic graphical model that performs probabilistic inference rather than identify the "hard" constraints that hold in spreadsheets. 


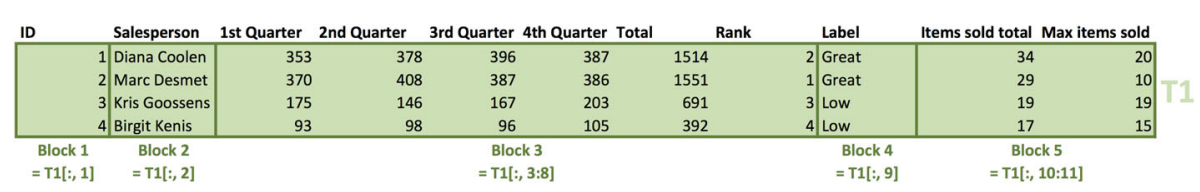

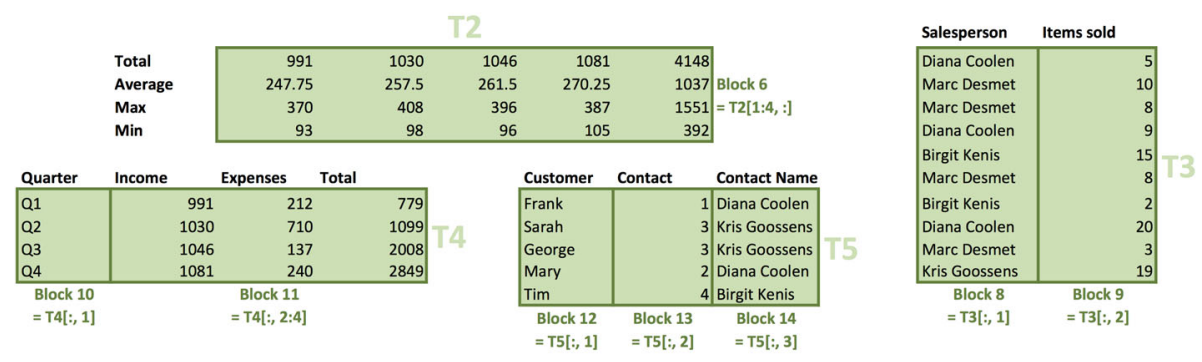

(a)

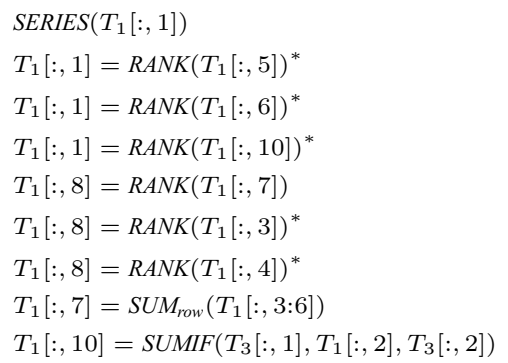

$$
\begin{aligned}
& T_{2}[1,:]=\operatorname{SUM}_{c o l}\left(T_{1}[:, 3: 7]\right) \\
& T_{2}[2,:]=\operatorname{AVERAGE} E_{c o l}\left(T_{1}[:, 3: 7]\right) \\
& T_{2}[3,:]=\operatorname{MAX}_{c o l}\left(T_{1}[:, 3: 7]\right) \\
& T_{2}[4,:]=\operatorname{MIN}_{c o l}\left(T_{1}[:, 3: 7]\right) \\
& T_{4}[:, 2]=\operatorname{SUM} M_{c o l}\left(T_{1}[:, 3: 6]\right) \\
& T_{4}[:, 4]=\operatorname{PREV}\left(T_{4}[:, 4]\right)+T_{4}[:, 2]-T_{4}[:, 3] \\
& T_{5}[:, 2]=\operatorname{LOOKUP}\left(T_{5}[:, 3], T_{1}[:, 2], T_{1}[:, 1]\right)^{*} \\
& T_{5}[:, 3]=\operatorname{LOOKUP}\left(T_{5}[:, 2], T_{1}[:, 1], T_{1}[:, 2]\right) \\
& T_{1}[:, 11]=\operatorname{MAXIF}\left(T_{3}[:, 1], T_{1}[:, 2], T_{3}[:, 2]\right)
\end{aligned}
$$

(b)

Fig. 1 Running example. a Example spreadsheet, black words and numbers only. Green background indicates headerless tables, dark borders indicate maximal type-consistent blocks. Most tables only contain typeconsistent columns while table $T_{2}$ also contains type-consistent rows. This example combines several Excel sheets based on exercises in the book "MS Excel 2010" (Van den Broeck and Cuypers 2011). b Constraints learned for the tables above, except 19 ALLDIFFERENT, 2 PERMUTATION and 5 FOREIGNKEY and 5 ASCENDING constraints not shown. Constraints marked with $*$ were not present in the original spreadsheets (Color figure online)

\section{Formalization}

Our goal is to automatically learn the constraints that hold between the rows and/or columns in a spreadsheet. This is applicable not just to data from spreadsheets, but to any data in tabular form.

We first introduce some terminology and the concept of a constraint template, after which we define the problem and discuss some additional considerations.

\subsection{Terminology}

Spreadsheets and tabular data may conceptually consist of multiple tables, as in Fig. 1a. Note that a table can contain one or more headers; however, we wish to reason over entire rows and columns of data, and hence we will consider headerless tables only. Headerless tables contain only data entries and are obtained by stripping away all contextual information such as headers. 
Formally, a (headerless) table is an $n \times m$ matrix. Each entry is called a cell. A cell has a type, which can be numeric or textual. We further distinguish numeric types in subtypes: integer and float. We also consider None as a special type when a cell is empty; None is a subtype of all other types.

A row or a column is type-consistent if all cells in that row or column are of the same base type, i.e., numeric or textual. We will use notation $T[a,:]$ to refer to the $a$-th row of table $T$ and similarly $T[:, a]$ for the $a$-th column. For example, in Fig. 1a, $T_{1}[:, 1]=[1,2,3,4]$ and $T_{3}[1,:]=$ ['Diana Coolen', 5]. $T_{3}[1,:]$ is not type-consistent while $T_{1}[:, 1]$ is.

The most important concept is that of a block.

Definition 1 A block has to satisfy three conditions: (1) it contains entire rows or entire columns of a single headerless table; (2) it is contiguous; and (3) it is type-consistent. The rows or columns have to be contiguous in the original table, meaning that they must visually form a block in the table, and each of the rows or columns has to be of the same type. If a block contains only rows we say it has row-orientation, if only columns, column-orientation.

In line with this definition, we can use the following notation to refer to blocks: $B=$ $T[a: b,:]$ for a row-oriented block containing rows $a$ to $b$ in table $T$; and similarly $B=$ $T[:, a: b]$ for a column-oriented block. We will refer to the vectors of a block when we wish to refer to its rows/columns independently of their orientation.

Blocks are used to reason about multiple rows or columns simultaneously. Requiring blocks to be contiguous is quite natural in the spreadsheet setting, as they logically group neighboring rows or columns. This matches the way people commonly construct formulas, by selecting a contiguous ranges in a sheet. For example, constraints such as summation often occur over contiguous blocks.

A block has the following properties:

- type: a block is type-consistent, so it has one type;

- table: the table that the block belongs to;

- orientation: either row-oriented or column-oriented;

- size: the number of vectors a block contains;

- length: the length of its vectors; as all vectors are from the same table, they always have the same length;

- rows: the number of rows in the block; in row-oriented blocks this is equivalent to the size;

- columns: the number of columns in the block; in row-oriented blocks this is equivalent to the length.

Example 1 Consider the (headerless) table $T_{1}$ in Fig. 1a. Its rows are not type consistent (i.e. they contain both numeric and textual data). However, the table can be partitioned into five column-oriented blocks $b_{1}, b_{2}, b_{3}, b_{4}, b_{5}$, as shown in the figure $\left(b_{1}=T_{1}[:, 1], b_{2}=T_{1}[:, 2]\right.$, $\left.b_{3}=T_{1}[:, 3: 8], \ldots\right)$.

Definition 2 Block containment $\sqsubseteq$. A block $B^{\prime}$ is contained in a block $B, B^{\prime} \sqsubseteq B$, iff both are valid blocks (contiguous, type consistent) with the same orientation and table, and each of the vectors in $B^{\prime}$ is also in $B$. For row-oriented blocks it means that: $B^{\prime} \sqsubseteq B \Leftrightarrow$ $B=T[a: b,:] \wedge B^{\prime}=T\left[a^{\prime}: b^{\prime},:\right] \wedge a \leq a^{\prime} \wedge b^{\prime} \leq b$ and similarly for column-oriented blocks.

We will sometimes write that $B^{\prime}$ is a subblock of $B$ or that $B$ is a superblock of $B^{\prime}$. An example of block containment is $T_{1}[:, 3: 6] \sqsubseteq T_{1}[:, 3: 8]$, which contains the sales numbers of all employees for the four quarters. 


\subsection{Constraint templates}

The goal is to learn constraints over blocks in the data. The knowledge needed to learn a constraint is expressed through constraint templates. A constraint template $s$ is a triple $s=($ Syntax, Signature, Definition $)$ :

- Syntax specifies the syntactic form of the constraint $s\left(B_{1}, \ldots, B_{n}\right)$, that is, the name of the template together with $n$ abstract arguments $B_{i}$; each of those arguments will correspond to a block. Thus, a constraint is viewed as a relation or predicate of arity $n$ in first order logic.

Functions can be represented in a similar way. Any function $B_{n+1}=s\left(B_{1}, \ldots, B_{n}\right)$ that has $n$ arguments and computes $B_{n+1}$ can be represented with an $(n+1)$-ary predicate $s^{\prime}\left(B_{1}, \ldots, B_{n}, B_{n+1}\right)$.

- Signature defines the requirements that the arguments of the predicate must satisfy. This can concern properties of individual blocks as well as relations between properties of arguments, for example, that the corresponding blocks must belong to the same table or have equal length. In terms of logical and relational learning (De Raedt 2008), the Signature is known as the bias of the learner, it specifies a conjunction of the properties that the arguments must have for the constraint to be well-formed. We denote this bias for a template $s$ using the predicate $\mathrm{Sig}_{s}$.

- Definition is the actual definition of the constraint that specifies when the constraint holds. Given an assignment of blocks to its arguments, it can be used to verify whether the constraint is satisfied or not by the actual data present in the blocks. In logical and relational learning this is known as the background knowledge. We introduce the predicate $D e f_{s}$ to capture this background knowledge for a template $s$.

The constraint templates implemented in TaCLe are defined in Table 1.

Example 2 Consider, for example, the constraint template for the row-based sum:

- Syntax: $B_{2}=S U M_{\text {row }}\left(\mathbf{B}_{1}\right)$, for arguments $B_{2}$ and $\mathbf{B}_{1}$.

- Signature: $B_{2}$ has to be a single vector $($ size $=1)$ while $\mathbf{B}_{\mathbf{1}}$ can be a block (size $\geq 1$ ), which can be derived from the use of a normal or bold font. Both blocks have to be numeric. This constraint is orientation-specific, so it requires that the number of rows in $\mathbf{B}_{1}$ equals the length of $B_{2}$. Moreover, the bias specifies that the number of columns to sum over is at least 2 . This avoids that $\mathbf{B}_{\mathbf{1}}$ contains only a single column.

- Definition: each value in the vector $B_{2}$ is obtained by summing over the corresponding row in $\mathbf{B}_{\mathbf{1}}$.

$S U M_{\text {row }}$ and $S U M_{\text {col }}$ are part of a family of constraints that we refer to as aggregate constraints that range over multiple rows or columns and are also available for aggregate operators MIN, MAX, AVERAGE, PRODUCT and COUNT. SUMIF is part of a family constraints that we refer to as conditional aggregate constraints which aggregate over a vector and only include those cells that satisfy a condition on a related cell in that table, e.g., for the spreadsheet in Fig. 1a: $T_{1}[:, 10]$ and the data in $T_{5}$ for matching salesperson names. Conditional aggregates are also available for the above aggregate operators, except for product. Note that SUMPRODUCT is not considered part of the family of aggregate constraints in this paper as it is not defined for other aggregate operators. See Sect. 4.4.3 for a more detailed discussion of the constraints used in TaCLe.

It is helpful to see the analogy of constraint templates with first order logic (FOL) and constraint satisfaction. From a FOL perspective, a constraint of the form $B_{2}=\operatorname{RANK}\left(B_{1}\right)$ 


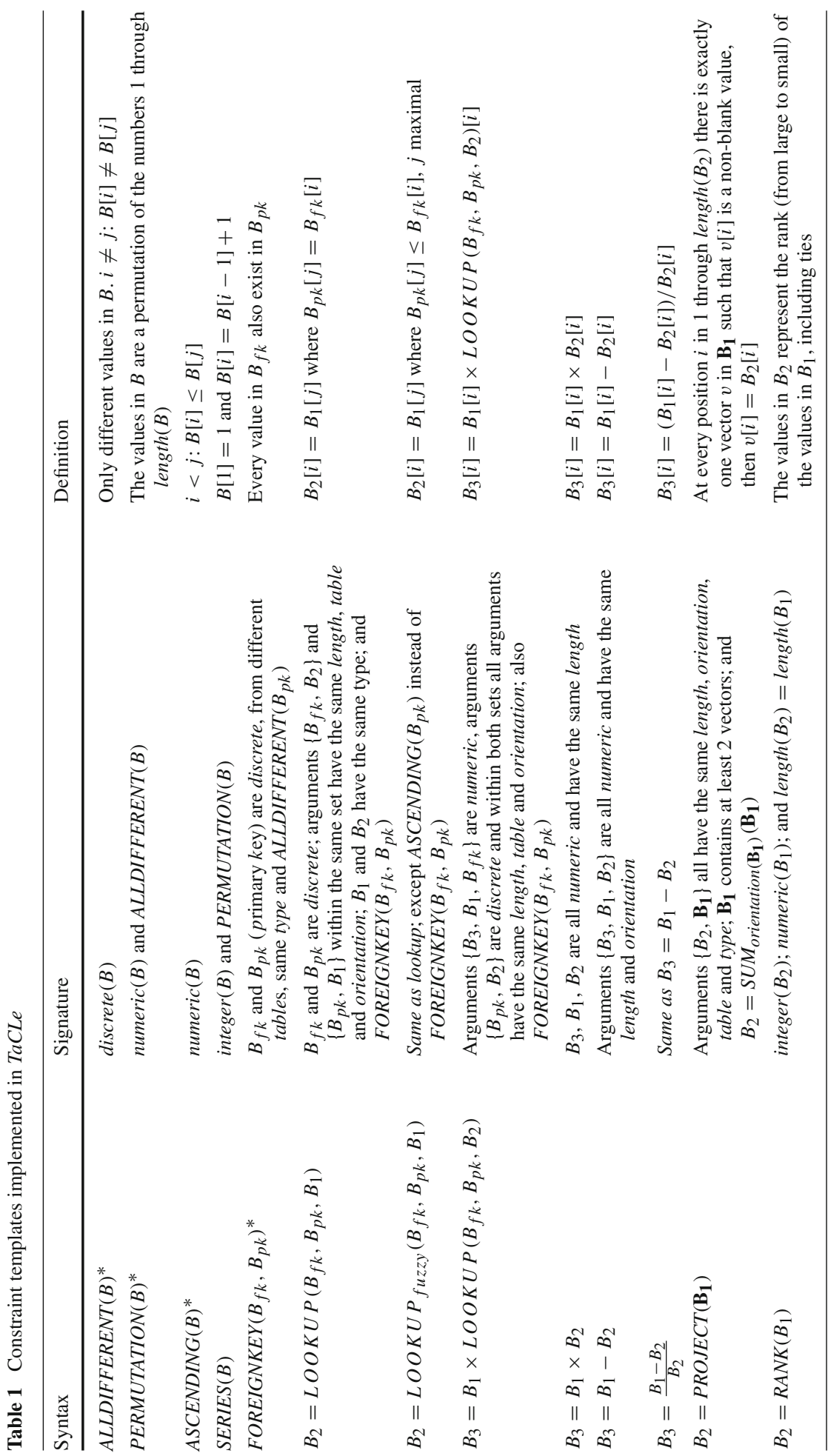




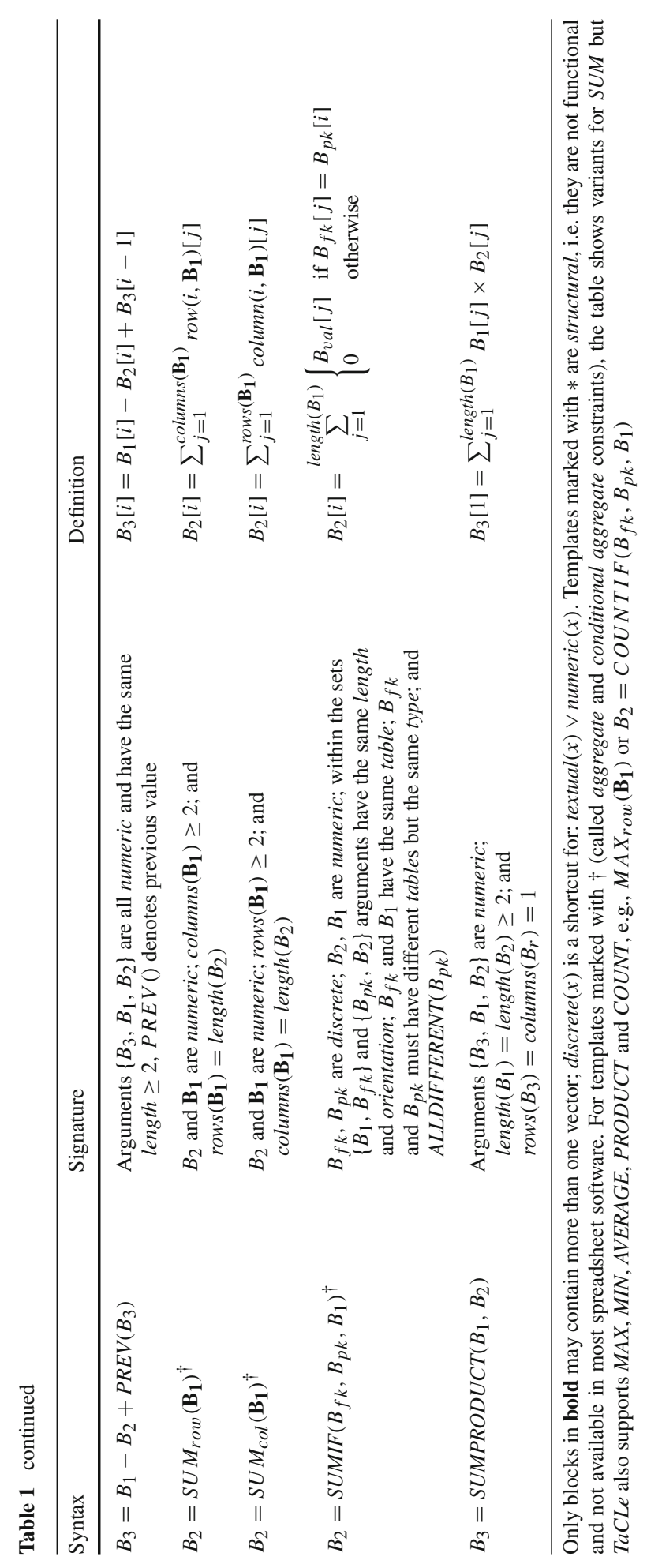


can be seen as a predicate $\operatorname{RANK}\left(B_{2}, B_{1}\right)$ where $R A N K$ is the name of the predicate and its arguments $B_{2}$ and $B_{1}$ are terms, which can be seen as either uninstantiated variables or as concrete values. This also holds in our setting, where an instantiation of a variable corresponds to a concrete block. For example, for the spreadsheet in Fig. 1a, when we write $T_{1}[:, 8]=\operatorname{RANK}\left(T_{1}[:, 7]\right)$, then the value of $B_{2}$ is the 8th vector in $T_{1}: B_{r}=T_{1}[:, 8]=$ $[2,1,3,4]$ and the value of $B_{1}$ is the 7th vector: $B_{x}=T_{1}[:, 7]=[1514,1551,691,392]$.

With this interpretation, we can speak about the signature and definition of a constraint template being satisfied. We say that a signature of a constraint template $s$ with $n$ arguments is satisfied by the blocks $\left(B_{1}, \ldots, B_{n}\right)$ if $\operatorname{Sig}_{s}\left(B_{1}, \ldots, B_{n}\right)$. Likewise a definition is satisfied if $\operatorname{Def}_{s}\left(B_{1}, \ldots, B_{n}\right)$ is. The template is hence satisfied if both the signature and definition are satisfied; in logic programming, we would define the predicate $s$ using a Prolog like clause: $s\left(B_{1}, \ldots, B_{n}\right) \leftarrow \operatorname{Sig}_{s}\left(B_{1}, \ldots, B_{n}\right) \wedge \operatorname{Def}_{s}\left(B_{1}, \ldots, B_{n}\right)$. Under this interpretation, the term constraint and constraint template can be used interchangeably.

Definition 3 A valid argument assignment of a constraint template $s$ is a tuple of blocks $\left(B_{1}, \ldots, B_{n}\right)$ such that $s\left(B_{1}, \ldots, B_{n}\right)$ is satisfied, that is, both the signature and the definition of the corresponding constraint template are satisfied by the assignment of $\left(B_{1}, \ldots, B_{n}\right)$ to the arguments.

\subsection{Problem definition}

The problem of learning constraints from tabular data can be seen as an inverse constraint satisfaction problem (CSP). In a CSP one is given a set of constraints over variables that must all be satisfied, and the goal is to find an instantiation of all the variables that satisfies these constraints. In the context of spreadsheets, the variables would represent blocks of cells, and one would be given the actual constraints and functions with the goal of finding the values in the cells. The inverse problem is, given only an instantiation of the cells, to find the constraints that are satisfied in the spreadsheet.

We define the inverse problem, that is the Tabular Constraint Learning Problem, as follows:

Definition 4 Tabular Constraint Learning.

Given: a set of instantiated blocks $\mathcal{B}$ over tables $\mathcal{T}$, and a set of constraint templates $\mathcal{S}$.

Find: all constraints $s\left(B_{1}^{\prime}, \ldots, B_{n}^{\prime}\right)$ where $s \in \mathcal{S}, \forall i: B_{i}^{\prime} \sqsubseteq B_{i} \in \mathcal{B}$ and $\left(B_{1}^{\prime}, \ldots, B_{n}^{\prime}\right)$ is a satisfied argument assignment of the template $s$.

The input is a set of blocks, and in Sect. 4 we will discuss how these can be extracted from a spreadsheet. Figure $1 \mathrm{~b}$ shows the solution to the tabular constraint learning problem when applied to the blocks of Fig. 1a and constraint templates listed in Table 1.

\subsection{Other considerations}

\subsubsection{Dependencies}

In Table 1 one can see that for some constraints we used the predicate of another constraint in its signature, e.g. for PERMUTATION. This expresses a dependency of the constraint on that other base constraint. This can be interpreted as follows: the signature of the constraint consists of its own signature plus the signature of the base constraint, and its definition of its own definition plus the definition of the base constraint. In FOL, we can see that one constraint entails the other, for example if PERMUTATION $(B)$ holds for a block $B$, then $\operatorname{ALLDIFFERENT(B)}$ also holds. 
While dependencies are optional, they simplify the specification of signatures and definitions. Moreover, in Sect. 4 we will see how such dependencies can be used to speed up the search for constraints.

\subsubsection{Redundancies}

Depending on the application, some constraints in the solution to the tabular constraint learning problem may be considered redundant. This is because constraints may be logically equivalent or may be implied by other constraints, e.g., if you know PERMUTATION $(B)$, then $A L L D I F F E R E N T(B)$ is redundant.

Within the same constraint, there can be equivalent argument assignments if the order of some of the arguments does not matter. For example, for the product constraint, $B_{3}=$ $B_{1} \times B_{2} \equiv B_{3}=B_{2} \times B_{1}$, so one can be considered redundant to the other. Two different constraints can also be logically equivalent, due to their nature, e.g., for addition / subtraction and product / division: $B_{3}=B_{1} \times B_{2} \equiv B_{1}=B_{3} / B_{2}$.

Finally, when the data has rows or columns that contain exactly the same values, then any constraint with such a vector in its argument assignment will also have an argument assignment with the other equivalent vectors.

Dealing with redundancy is often application-dependent. Therefore, in Sect. 4 we first explain our generic method for finding all constraints, before describing some optimizations that avoid obvious equivalences (Sect. 4.4). In the experiments we will investigate the impact of redundancy further.

\section{Approach to tabular constraint learning}

The aim of our method is to detect constraints between rows and columns of tabular data. Recall that a valid argument assignment for a constraint is an assignment of a block to each of the arguments of the constraint, such that the signature and definition of the constraint template is satisfied.

Our proposed methodology contains the following steps (Algorithm 1):

\footnotetext{
Algorithm 1 The TaCLe approach

Step 1 Extract headerless tables from tabular data

Step 2 Partition the tables into contiguous, type-consistent blocks (input blocks) using the BlockDetect tool

Step 3 Generate for each constraint template all valid argument assignments in two steps:

(a) For each constraint template $s$, generate all assignments $\left(B_{1}, \ldots, B_{n}\right)$ where each $B_{i}$ is an input block and the $B_{i}$ are compatible (defined below) with the signature of $s$.

(b) For each generated assignment $\left(B_{1}, \ldots, B_{n}\right)$, find all constraints $s\left(B_{1}^{\prime}, \ldots, B_{n}^{\prime}\right)$ such that $B_{i}^{\prime} \sqsubseteq B_{i}$ holds for all $i$ and the signature and definition of $s$ are satisfied.
}

The core of our method is step 3. In principle, one could use a generate-and-test approach by generating all possible blocks from the input blocks and testing each combination of blocks for each of the arguments of the constraints. However, each input block of size $m$ has $m *(m+1) / 2$ contiguous subblocks, meaning that a constraint with $n$ arguments would have to check $O\left(n^{m^{2}}\right)$ combinations of blocks. 
Instead, we divide this step into two parts: in step 3a, we will not reason over individual (sub)blocks and their data, but rather over the properties of the input blocks. Consider table $T_{1}$ in Fig. 1a and the $S U M_{\text {row }}$ constraint template. Instead of considering all possible subblocks of $T_{1}\left(b_{1}, b_{2}, b_{3}, b_{4}, b_{5}\right)$, we first reason over the properties of the input blocks. For example, blocks $b_{2}$ and $b_{4}$ are not numeric so they can be immediately discarded. Input blocks $b_{1}, b_{3}$ and $b_{5}$ are valid candidates for the second argument of the constraint, i.e., the functionally defined block. However, since they all have length 4, the first argument, which is the block to sum over, needs at least 4 columns which is only satisfied by $b_{3}$. Hence, the assignments generated for $S U M_{\text {row }}$ in step 3 a would be $\left(b_{1}, b_{3}\right),\left(b_{3}, b_{3}\right),\left(b_{5}, b_{3}\right)$.

In step $3 \mathrm{~b}$, we start from an assignment $\left(B_{1}, \ldots, B_{n}\right)$ with input blocks $B_{i}$ and generate and test all possible (sub)block assignments to the arguments, using the properties and actual data of the blocks. As we only have to consider the blocks $B_{i}^{\prime} \sqsubseteq B_{i}$ contained in each input blocks, this is typically an easier problem to solve. For $S U M_{\text {row }}$ in the above example and assignment $\left(b_{5}, b_{3}\right)$, each of the vectors of $b_{5}$ will be considered as candidate for the left-hand side, and one can enumerate all subblocks of $b_{3}$ for the right-hand side, verify the signature (e.g. at least size 4) and test whether for each row the definition is satisfied.

We now describe in more detail how headerless tables are extracted (step 1, Sect. 4.1), how input blocks are generated from them (step 2, Sect. 4.2), and in Sect. 4.3 how the candidate input block assignments are generated (Step 3a) and how the actual assignments are extracted from that (Step 3b). In Sect. 4.4 we describe a number of optimizations designed to improve the effectiveness algorithm.

\subsection{Step 1: Table extraction}

Many spreadsheets contain headers that provide hints at where the table(s) in the sheet are and what the meaning of the rows and columns is. However, detecting tables and headers is a complex problem on its own (Fang et al. 2012). Furthermore, headers may be missing and their use often requires incorporating language-specific vocabulary, e.g. English.

Instead, our algorithm will assume tables are specified by means of their coordinates within a spreadsheet and optionally a fixed orientation of each table. The orientation can be used to indicate that a table should be interpreted as having only rows or only columns.

We developed two simple tools that can be used for the specification of the tables:

Automatic detection (AutoExtract) Under the following two assumptions, the table detection task becomes easy enough to be automated; 1) tables are rectangular blocks of data not containing any empty cells; and 2) tables are separated by at least one empty cell from other tables. The sheet is then processed row by row, splitting each row into ranges of non-empty cells and appending them to adjacent ranges in the previous row. Headers can be detected, for example, by checking if the first row or column contains only textual values. If a header row (column) is detected, it is removed from the table and the orientation of the table is fixed to columns (rows), otherwise, we assume there is no header and the orientation is not fixed. Our implementation of this approach is called the AutoExtract tool.

Visual selection (VisualExtract) The above assumptions do not hold for many tables. However, since the specification of tables is usually easy for humans, we employ a second approach which allows users to indicate tables using a visual tool. Screenshots are available in the accompanying GitHub repository with meta-data ${ }^{1}$ and in "Appendix 2". Users select tables excluding the header and optionally specify an orientation. The VisualExtract tool then generates the specification of the tables automatically.

\footnotetext{
${ }^{1}$ https://github.com/SergeyParamonov/TaCLe.
} 


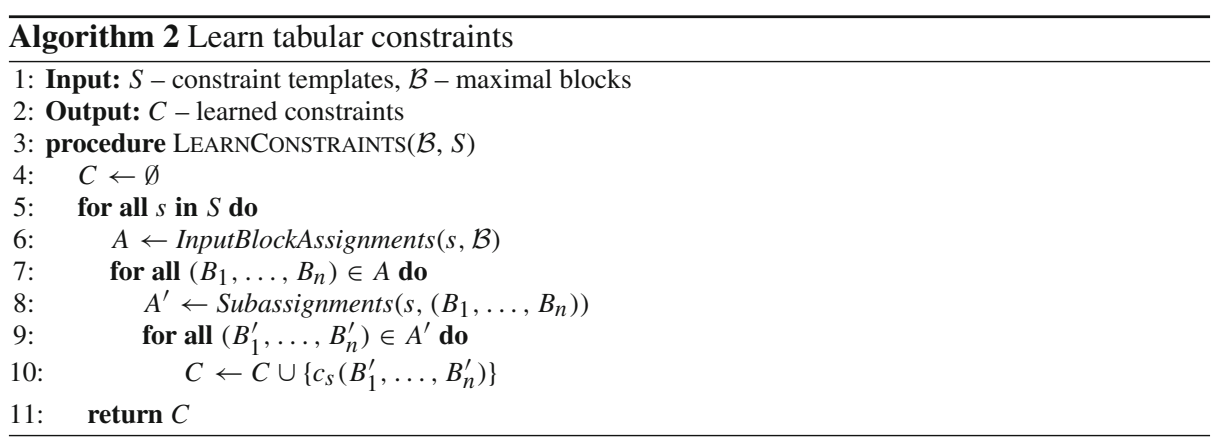

\subsection{Step 2: Block detection with BlockDetect}

The goal of the block detection step is to partition tables into maximally type-consistent (all-numeric or all-textual) blocks. First, the BlockDetect tool preprocesses the spreadsheet data so that currency values and percentual values are transformed into their corresponding numeric (i.e. integer or floating point) representation (e.g. $2.75 \$$ as 2.75 and $85 \%$ as 0.85 ). Then, each table is partitioned into maximal type-consistent blocks.

To find row-blocks, each row is treated as a vector and must be type-consistent; similarly for columns and column-blocks. Then, adjacent vectors that are of the same type are merged to obtain the maximally type-consistent blocks.

\subsection{Step 3: Constraint learning algorithm}

Our learning method assumes that constraint templates and input blocks are given and solves the Tabular Constraint Learning problem by checking for each template: what combination of input blocks can satisfy the signature (input block assignment) and which specific subblock assignments satisfy both the signature and the definition. The pseudo-code of this approach is shown in Algorithm 2.

This separation of checking the properties of input block assignments from checking the actual data in the subblock assignment controls the exponential blow-up of combinations to test. Furthermore, we use constraint satisfaction technology in the first step to efficiently enumerate input block assignments that are compatible with the signature.

\section{Step 3a: Generating input block assignments}

Given a constraint template $s$ and the set of all input blocks $\mathcal{B}$, the goal is to find all combinations of input blocks that are compatible with the constraint signature. An argument assignment $\left(B_{1}, \ldots B_{n}\right)$ is compatible with the signature of template $s$ if for each block $B_{i}$ there exists at least one subblock $B_{i}^{\prime} \sqsubseteq B_{i}$ that satisfies the signature.

The choice of one argument can influence the possible candidate blocks for the other arguments, for example, if they must have the same length. Instead of writing specialized code to generate and test the input block assignments, we make use of the built-in reasoning mechanisms of constraint satisfaction solvers.

A Constraint Satisfaction Problem (CSP) is a triple $(V, D, C)$ where $V$ is a set of variables, $D$ the domain of possible values each variable can take and $C$ the set of constraints over $V$ that must be satisfied. In our case, we define one variable $V_{i}$ for each argument of a constraint 
Table 2 Translation of signature requirements to input block constraints. The following need not be relaxed: length $(B)=x$, orientation $(B)=x, \operatorname{table}(B)=x$ and $\operatorname{size}(B) \geq x$

\begin{tabular}{ll}
\hline Requirement & Input block constraint \\
\hline $\operatorname{type}(B)=x$ & basetype $($ type $(B))=$ basetype $(x)$ \\
$\operatorname{size}(B)=x$ & $\operatorname{size}(B) \geq x$ \\
$\operatorname{columns}(B)=x$ & if orientation $(B)=\operatorname{column}: \operatorname{columns}(B) \geq x$ \\
& if orientation $(B)=\operatorname{row}: \operatorname{columns}(B)=x$ \\
$\operatorname{rows}(B)=x$ & if orientation $(B)=\operatorname{column}: \operatorname{rows}(B)=x$ \\
& if orientation $(B)=\operatorname{row}: \operatorname{rows}(B) \geq x$ \\
\hline
\end{tabular}

template. Each variable can be assigned to one of the input blocks in $\mathcal{B}$, so the domain of the variables consists of $|\mathcal{B}|$ block identifiers.

To reason over the blocks, we add to the constraint program a set of background facts that contain the properties of each input block, namely its type, table, orientation, size, length and number of rows and columns.

The actual CSP constraints must enforce that an input block assignment is compatible with the requirements defined in the signature. Table 2 shows how the conversion from requirements of the signature to CSP constraints works: Requirements on the lengths, orientations and tables of blocks can be directly enforced since they are invariant under block containment (ㄷ). Typing requirements need to be relaxed to check only for base types, namely numeric and textual. Minimum sizes can be directly enforced, but exact size requirements are relaxed to minimum sizes, since blocks with too many vectors contain subblocks of the required smaller size. Finally, restrictions on the number of rows or columns behave as length or size constraints based on the orientation of the block they are applied to.

The InputBlockAssignments $(s, \mathcal{B})$ method will use these conversion rules to construct a CSP and query a solver for all solutions. These solutions correspond to the valid input block assignments for constraint template $s$.

Example 3 Consider the constraint template $B_{2}=S U M_{\text {row }}\left(\mathbf{B}_{1}\right)$, then the generated CSP will contain two variables $V_{2}, V_{1}$ corresponding to arguments $B_{2}$ and $\mathbf{B}_{\mathbf{1}}$. Given maximal blocks $\mathcal{B}$, the domain of these variables are $D\left(V_{1}\right)=D\left(V_{2}\right)=\{1, \ldots,|\mathcal{B}|\}$. Finally, the signature of $B_{2}=S U M_{\text {row }}\left(\mathbf{B}_{1}\right)$ (see Table 1) will be translated into constraints:

$$
\begin{aligned}
& \operatorname{numeric}\left(V_{2}\right) \wedge \text { numeric }\left(V_{1}\right) \wedge \operatorname{columns}\left(V_{1}\right) \geq 2 \\
& \left(\operatorname{orientation}\left(V_{1}\right)=\operatorname{column}\right) \Rightarrow\left(\operatorname{rows}\left(V_{1}\right) \geq \operatorname{length}\left(V_{2}\right)\right) \\
& \left(\operatorname{orientation}\left(V_{1}\right)=\operatorname{row}\right) \Rightarrow\left(\operatorname{rows}\left(V_{1}\right)=\operatorname{length}\left(V_{2}\right)\right)
\end{aligned}
$$

\section{Step 3b: Generating subblock assignments}

Given an input block assignment $\left(B_{1}, \ldots, B_{n}\right)$ from the previous step, the goal is to discover valid subassignments, i.e., assignments of subblocks $\left(B_{1}^{\prime}, \ldots, B_{n}^{\prime}\right)$ with for all $i, B_{i}^{\prime} \sqsubseteq B_{i}$, that satisfy both the signature and the definition of template $s$.

Example 4 Consider the sum-over-rows template $B_{2}=S U M_{\text {row }}\left(\mathbf{B}_{1}\right)$ again; an example input block assignment from Fig. 1a is $\left(B_{2}, \mathbf{B}_{1}\right)=\left(T_{1}[:, 3: 8], T_{1}[:, 3: 8]\right)$. Note that this assignment does not satisfy the signature yet, because $B_{2}$ contains more than one vector. This step aims to generate subassignments $\left(B_{2}^{\prime} \sqsubseteq B_{2}, \mathbf{B}_{1}{ }^{\prime} \sqsubseteq \mathbf{B}_{1}\right)$ that do satisfy the signature 
and test whether they satisfy the definition: $\forall i, B_{2}[i]=\sum_{j=1}^{\text {length }\left(\mathbf{B}_{1}\right)} \operatorname{row}\left(i, \mathbf{B}_{\mathbf{1}}\right)[j]$. In Fig. 1a there is exactly one such subassignment: $\left(T_{1}[:, 7], T_{1}[:, 3: 6]\right)$.

In this step, we could also formulate the problem as a CSP. However, few CSP solvers support floating point numbers, which are prevalent in spreadsheets. Furthermore, in a CSP approach we would have to ground out the definition for each of the corresponding elements in the vectors. This is inefficient, as such blocks may not even satisfy the signature or just the first element in the data may already not satisfy the definition.

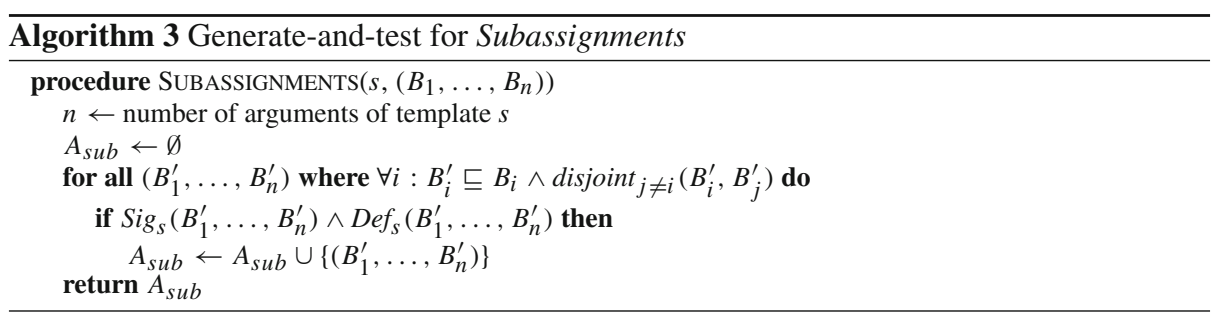

On the other hand, a simple generate-and-test approach, as illustrated in Algorithm 3, will usually suffice. Given an input block assignment, all disjoint subassignments are generated taking into account the required size, columns or rows. For every disjoint subassignment the exact signature (e.g. subtypes will not have been checked yet) and definition will be tested and all satisfying subassignments are returned.

Based on the assumptions that most subassignments will not satisfy the definition of the constraint template, the implementation of the definition check is geared to fail-fast when possible. Specifically, for many constraints the entries of every subblock are fetched one by one, and as soon as one does not hold failure is returned. Often, the first entry will already not satisfy the definition (e.g. if $B_{3}[0] \neq B_{1}[0] \times B_{2}[0]$ then $B_{3}=B_{1} \times B_{2}$ does not hold). As a result, many subassignments can be discarded by looking at just one or a few entries, and considering all subassignments that will be checked, the runtime of the checks will typically not be influenced much by the length of the vectors.

\subsection{Optimizations}

This section discusses various design decisions and optimizations aimed at improving the extensibility and effectiveness of our method, as well as reducing the redundancy in the output.

\subsubsection{Template dependencies}

As discussed in Sect. 3.4.1, some constraint templates depend on others by including templates they depend on in their signature (see Table 1).

In Inductive Logic Programming, one often exploits implications between constraints to structure the search space (De Raedt 2008). Our approach uses the dependencies between templates to define a dependency graph. Dependencies are provided to the system as part of the specification of the constraint templates. We assume that signatures do not contain equivalences or loops, and hence the resulting graph is a directed acyclic graph (DAG). Figure 2 shows the dependency graph extracted from the signatures in Table 1. Constraint templates that have no dependencies are omitted. 


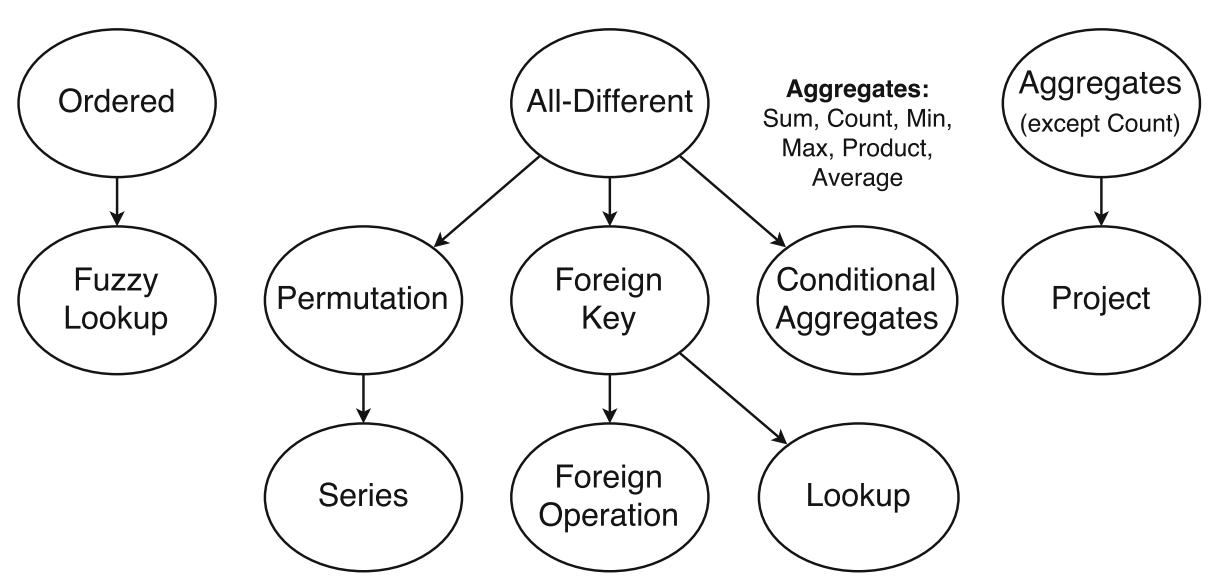

Fig. 2 Dependency graph of Table 1; an arrow from $s_{1}$ to $s_{2}$ indicates that $s_{2}$ depends on $s_{1}$ : its signature includes $s_{1}$

Using the dependency graph $(\mathcal{D})$ we can reorder the templates such that a template occurs after any template it depends on. Concretely, in Algorithm 2 line 5 the input templates $S$ are handled following an ordering that agrees with the partial ordering imposed by $\mathcal{D}$ (e.g. ALLDIFFERENT before FOREIGNKEY). When using this ordering, templates later in the ordering can use the learned constraints of the templates they depend on. To enable this reuse of constraints, the set of learned constraints $C$ is added to the argument of InputBlockAssignments on line 6.

The previously learned constraints are used to speed up the InputBlockAssignments step. For constraint templates where some arguments are also part of a constraint that it depends on, it is not needed to search for input blocks and subblocks from scratch. Instead, one can start from all and only the actual argument assignments of the base constraint, and only search matching input blocks for the remaining arguments. Therefore, the number of candidate assignments for a constraint template can be reduced by adding a dependence on a lower arity constraint.

Example 5 Consider FOREIGNKEY $\left(B_{f k}, B_{p k}\right)$, which states that every value in $B_{f k}$ also exists in $B_{p k}$; its signature includes ALLDIFFERENT $\left(B_{p k}\right)$. There are 18 ALLDIFFERENT constraints to be found in Fig. 1a, hence, the InputBlockAssignments only needs to check which input blocks for $B_{f k}$ are compatible (different table, same type) with these 18 assignments to $B_{p k}$.

In this case, instead of generating one CSP to find all assignments, a CSP is generated for every known assignment of the depending constraint, which then searches for all assignments completing this partial assignment. The procedure to generate CSPs remains the same otherwise.

\subsubsection{Redundancy}

We consider two types of redundancy that we aim to eliminate during search. Specifically, these redundancies are taken into account in the implementation for finding subassignments for a constraint. As noted in Sect. 3.4.1, for some constraint templates there are symmetries over the arguments that lead to trivially equivalent solutions, for example, $B_{3}^{\prime}=B_{1}^{\prime} \times B_{2}^{\prime} \Leftrightarrow$ 
$B_{3}^{\prime}=B_{2}^{\prime} \times B_{1}^{\prime}$. Such duplicates are avoided by defining a canonical form for these constraints. In practice, we define an arbitrary but fixed block ordering and require those blocks that are interchangeable to adhere to this ordering. Moreover, among the semantically equivalent constraints product $\left(B_{3}^{\prime}=B_{1}^{\prime} \times B_{2}^{\prime}\right)$ and division $\left(B_{1}^{\prime}=B_{3}^{\prime} / B_{2}^{\prime}\right)$ we only added product, as well as only difference $\left(B_{3}=B_{1}-B_{2}\right)$ and not sum $\left(B_{1}=B_{3}+B_{2}\right)$. However, division and sum could easily be added, and they could equivalently be added in post-processing based on the matching product and difference constraints.

A last type of redundancy we chose to avoid during search is that there may be multiple overlapping subblocks that satisfy the definition of a constraint template. For example, consider the constraint $B_{2}=S U M_{c o l}(T[:, 1: n])$ where $T[:, k: n]$ consists of only zeros, then $B_{2}=S U M_{c o l}(T[:, 1: j])$ will be true for all $k-1 \leq j \leq n$. In TaCLe we have, therefore, chosen to only consider maximal subblocks. This can be seen as a bias of our system.

In some cases a maximal subblock might falsely include irrelevant columns. Consider input block $\mathbf{B}_{1}=T[:, 1: 3]=\left[[200,300]\right.$, [200, 150], [1, 2]] and $B_{2}=[200,300]$, then TaCLe will find the constraint $B_{2}=\operatorname{MAX}(T[:, 1: 3])$. Should the target constraint be $B_{2}=$ $\operatorname{MAX}(T[:, 1: 2])$, then TaCLe is only able to find exactly that constraint if $T[:, 3]$ is split off into a separate block in the block detection phase or by post-processing the detected constraints.

\subsubsection{Constraints}

The 33 constraints that are currently supported in our system are shown in Table 1 . We included most formulas that we encountered in tutorial spreadsheets including the popular SUM and LOOKUP constraints. ${ }^{2}$ We also added four structural constraints (ALLDIFFERENT, PERMUTATION, ASCENDING and FOREIGNKEY) so that they can be used in the signature of other constraint templates; they are also popular in constraint satisfaction (Beldiceanu and Simonis 2012). In our current system, the signature for these structural constraints was chosen to be strict, i.e. only support those types that are required by depending constraints. Therefore, ALLDIFFERENT does not support floating point numbers and ASCENDING does not support strings.

For LOOKUP, aggregate constraints and PROJECT we use optimized implementations to find subassignments instead of generic generate-and-test.

Given an input block assignment, the implementation for $B_{2}=L O O K U P\left(B_{f k}, B_{p k}, B_{1}\right)$ first generates candidate vectors for $B_{p k}$ and $B_{1}$, then populates a hash table that allows it to quickly look up keys, afterwards it generates candidate vectors for the remaining arguments and, finally, checks which subassignments are valid.

For aggregates (e.g. SUM) and PROJECT, a custom implementation is used that tries to find maximal subblocks that satisfy the constraint templates. Instead of generating all possible subblocks, given an inputblock assignment, these implementations start from an input block and repeatedly generate and test smaller subblocks. Therefore, once a match is found, all smaller subblocks do not need to be considered anymore.

Finally, for FOREIGNKEY and conditional aggregates, generate-and-test is used, but in combination with caches that store intermediate results and a preparation step to populate some of the caches. For example, for conditional aggregates, input block assignments are analyzed to find vectors with overlapping values. These results are then cached and used to quickly reject subassignments where there is no overlap between $B_{p k}$ and $B_{f k}$.

2 https://support.office.com/en-us/article/Excel-functions-by-category-5F91F4E9-7B42-46D2-9BD1-63F26 A86C0EB. 


\subsubsection{Limited precision}

One of the challenges of discovering mathematical functional constraints, such as product, is that values in spreadsheets often have limited precision and might have been rounded. Testing whether such a constraint holds for a given subassignment, e.g., $B_{3}=B_{1} \times B_{2}$, can be done by calculating the expected result $\left(B_{1} \times B_{2}\right)$ first and testing if it corresponds to the actual result $\left(B_{3}\right)$. For the values $B_{1}=$ [1.7], $B_{2}=$ [1.8], $B_{3}=$ [3.1], we would compute first $B_{1} \times B_{2}=[3.06]$ and conclude [3.06] $\neq$ [3.1] unless the expected result is first rounded. Therefore, TaCLe analyzes how long the fractional part of an actual result is and rounds the expected result accordingly.

\section{Evaluation}

In this section we experimentally validate our approach. We first explain the experimental setup and illustrate it on the example in Fig. 1a, we then investigate the effectiveness of our method on synthetic spreadsheets, after which we evaluate our method on spreadsheets from various sources.

\subsection{Experimental setup}

All spreadsheets used in the experiments are in CSV format and for each spreadsheet the tables are specified. The tables were obtained by running the table extraction tool, AutoExtract. Manual intervention of the simple automatic detection was needed only in the case of None values (e.g. to merge split-up tables caused by the None's) and in cases where the headers are ambiguous (e.g. headers containing textual and numeric cells). In these cases the tables specifications were generated using the VisualExtract tool.

Blocks are then detected automatically using the block detection algorithm, BlockDetect. Manual intervention was required for tables that contain None values (e.g. if the type of empty cells cannot be inferred).

All spreadsheets also have a set of ground-truth of constraints, we call these the intended constraints, that are expected to be (re-)discovered. These were determined by us using either the formulas of the original sheet if present, or inferring them based on, for example, the header names in the sheet. Five spreadsheets contained rows or columns that were entirely identical in which case results can be computed in multiple ways and there are multiple valid constraints. For these spreadsheets, we used the original formulas when present and otherwise inferred the intended constraints based on the headers and the location within the sheet. However, some of the intended constraints are currently outside of the scope of TaCLe, in particular nested mathematical or nested logical formulas. We denote by supported constraints the subset of intended constraints that the system can find in theory.

In the following experiments, we focus on spreadsheets specifically and hence only include functional constraints, constraints that can be expressed as formulas. Therefore, we filter out the structural constraints ALLDIFFERENT, FOREIGNKEY, PERMUTATION and ASCEND$I N G$ in the output. All constraints are stored in their canonical form.

We will use recall and precision to measure how well our tool is performing. Recall is the fraction of intended constraints actually discovered by the system: $\frac{\text { intended discovered }}{\text { all intended }}$ while precision is the fraction of constraints found by the system that are indeed intended: intended discovered.

all discovered 
We include a no-CSP baseline to compare our approach to. This baseline follows a similar approach as TaCLe, however, it does not prune input blocks in step 3a and generates subassignments directly. Therefore, the signature is also only tested on the subassignment level. While the modified approach still benefits from the reuse of earlier solutions, it does not generate partial assignments for dependent constraint templates.

All experiments were run using Python 3.5 .1 on a Macbook Pro, Intel Core i7 $2.3 \mathrm{GHz}$ with 16 GB RAM. The constraint solver that is used by the input block assignment phase (Step 3a) is python-constraint (Niemeyer).

\subsection{Results on the running example}

For the example presented in Fig. 1a, TaCLe takes a few seconds to find the constraints listed in Fig. 1b. These include 5 spurious $R A N K$ constraints, e.g. $T_{1}[:, 1]=R A N K\left(T_{1}[:, 5]\right)$, that are true by accident. Moreover, there is one LOOKUP constraint that was not intended in the original spreadsheet (looking up ID based on Salesperson) and which is symmetric to the intended one (Salesperson based on ID).

Hence, for this example we achieve a recall of $100 \%$, that is, all intended constraints are discovered, and a precision of $12 / 18=67 \%$. All intended constraints are supported. The recall is perfect, but the precision is rather low. This is mostly due to the spurious RANK constraints. We note that the tables are quite short, which increases the chance of a constraint like $R A N K$ to be true by chance; we expect less spurious constraints on larger datasets.

We now investigate three main questions that influence the quality of the solutions found by a constraint learning method:

- $Q_{1}$ : the method may fail to find intended constraints if it takes too much time to find them; what are the factors that influence the runtime of the method most?

- $Q_{2}$ : the method may fail to find intended constraints because it does not support such constraints; how does our method perform on real spreadsheets?

- $Q_{3}$ : the method may find non-intended constraints; how many and what type of nonintended constraints are found on real spreadsheets?

We first investigate $Q_{1}$ on synthetic spreadsheets and investigate $Q_{1}, Q_{2}$ and $Q_{3}$ on a collection of real spreadsheets.

\subsection{Effectiveness on synthetic data}

In this section we investigate the factors that influence the runtime of TaCLe the most; we do this by investigating the impact on the running time of 1) the number of vectors; 2) the length of the vectors; and 3) the size of the blocks. This analysis is accomplished by testing our system on synthetic spreadsheets. The timeout for all experiments was set to 200 seconds.

Spreadsheet generation The synthetic sheets should trigger the checking of many different constraints, both in the first phase (input block assignment) and in the second phase (subblock assignment), in order to clearly study the effect on the constraint checking. As many constraints require numeric or discrete data, we will use integers in the synthetic sheets as these are both numeric and discrete.

We generate spreadsheets with random data. The result is that there are no intended constraints in the data. This is reasonable, as even in real data most of the constraints will not hold. Additionally, for vectors in random data the ALLDIFFERENT constraint is likely to hold, which is a requirement for many other constraint templates. 


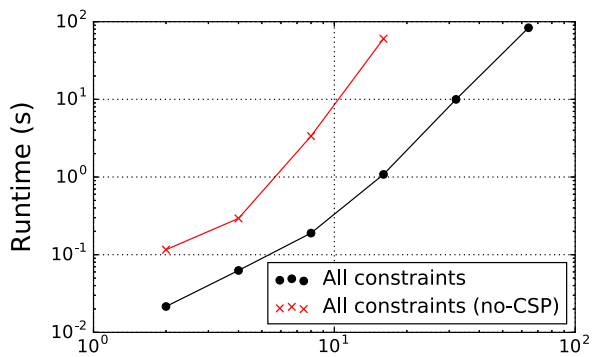

(a)

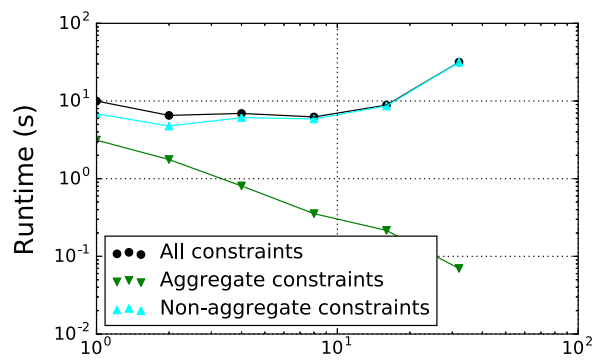

(c)

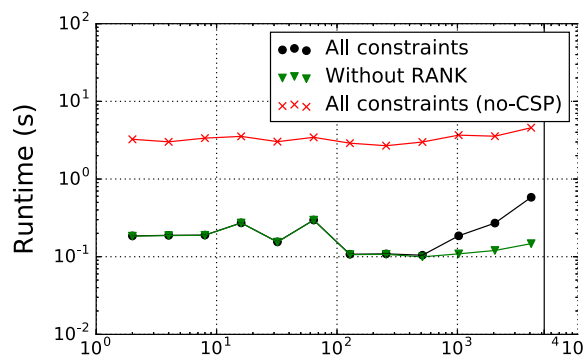

(b)

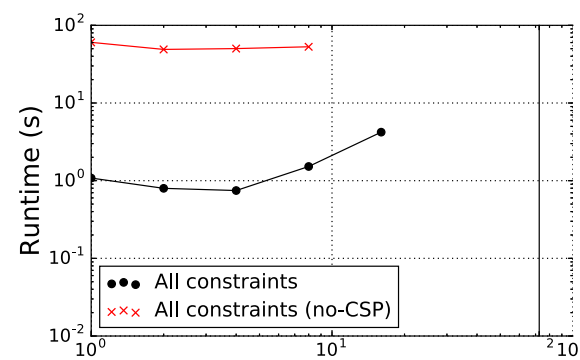

(d)

Fig. 3 Loglog plots of the experiments with synthetic (random) data. In plot $a$ the number of vectors is increased; in plot $b$ the size of the vectors is increased; and in plots $c$ and $d$ the number of blocks is increased (the block size is decreased). Missing values for the no-CSP baseline indicate that it timed out. a Number of vectors. b Vector size. c Number of blocks (32 vectors). d Number of blocks (16 vectors)

The synthetic spreadsheets contain two tables, because a number of constraints require that their arguments are picked from different tables. For one table the number of vectors and block size is fixed to two, while for the other table these properties are varied according to the experimental setup. The reported number of vectors and the block size in the experiments refer to those of the second table. The length of the vectors can also be adapted and applies to both tables, since equal length vectors are a required property of many constraints. Unless otherwise mentioned, the 'adaptable' table consists of one block of 8 vectors of length 8 , and hence 2 vectors of length 8 in the 'fixed' table.

Effect of number of vectors Figure 3a shows the results of our experiments, in which TaCLe was run on synthetic spreadsheets containing an increasing number of equally sized vectors. As shown in the figure, the number of vectors in a spreadsheet can have a significant impact on the running time. Moreover, TaCLe performs favorably to the no-CSP baseline whose running time not only starts off slower but also grows faster.

The number of vectors is expected to impact the running times, especially when few input block assignments can be discarded in the first phase. This occurs, for example, when most vectors belong to the same block or all vectors have the same type and size as we artificially enforced in this experiment. In this case, for a constraint template that requires $n$ single vector arguments and a spreadsheet with $v$ vectors, up to $\frac{v !}{(v-n) !}$ subassignments are generated and checked by the algorithm.

Typically, we expect the number of generated subassignments to be much smaller in real world spreadsheets and, therefore, the running time to be less sensitive to the number of 
vectors. This is because in the first phase entire blocks can be disqualified that do not match the signature requirements, e.g. blocks of vectors with different lengths.

Effect of length of vectors To measure the effect that the length of the vectors has on the running time, we ran our system on spreadsheets in which the length of the vectors was gradually increased. Figure $3 \mathrm{~b}$ summarizes our results and shows that the length of the vectors has a small impact on the performance for most templates. The no-CSP baseline performs similarly, however, its runtime is higher by a large margin.

As discussed in Sect. 4.4.3, our implementation attempts to detect as fast as possible if a subassignment does not satisfy a constraint template. Our experiments satisfy the assumption that the large majority of possible subassignments do not satisfy most constraints. Therefore, the results confirm that our implementation is able to severely limit the impact of the length of vectors.

Most of the increase in running time can be attributed to the RANK constraint. The implementation is often not able to quickly detect that subassignments do not satisfy RANK, meaning that it will have to analyze many if not all entries in the vector.

Effect of block size To measure the effect of changing the block size, the number of vectors is set to 32 and the vectors are explicitly split into varying number of blocks/tables (up to 32). Figure $3 \mathrm{c}$ shows the running times of TaCLe for various settings. As the blocks become smaller, the total running time first decreases before increasing again. Looking at the running times for aggregate constraints and non-aggregate constraints separately reveals that they are affected differently by the block size. For aggregate constraints (green line) the running time decreases with the block size, while for non-aggregate constraints (red line) the running time first stays more or less constant but then increases more strongly as the size of the blocks becomes small.

Aggregate constraints, e.g. sum or max, have an argument that allows subblocks of varying sizes while non-aggregates only allow single vector arguments. Hence, for aggregate constraints our method will try to find any subblock in the given input block that satisfies the constraint. Since a input block of size $m$ has $m(m+1) / 2$ contiguous subblocks, the search for aggregate constraints becomes faster if there are more but smaller blocks. ${ }^{3}$

Non-aggregate constraints have only single vector arguments. By splitting a set of vectors into many blocks, however, there will be a larger number of input blocks given as input to the first phase. In the synthetic spreadsheets, the first phase, which looks at properties of input blocks alone, can disqualify few or no input block assignments, leading to some increase in runtime as more blocks are added.

In order to compare to the baseline, we had to lower the number of vectors from 32 to 16. Figure $3 \mathrm{~d}$ shows that here, too, our system performs better. Both approaches profit from lower running times for aggregates and the no-CSP baseline can offset some of the slowdown as the block size grows small.

These experiments with synthetic data have given us a better understanding of what effect the properties of vectors and blocks can have on runtime and hence on the ability of the algorithm to finish within reasonable time. We next investigate the behavior of our method on real spreadsheets.

${ }^{3}$ Consider, for example, $v$ vectors distributed over $b$ blocks of size $v / b$, the number of subblock candidates then is $O\left(b * v^{2} / b^{2}\right)=O\left(v^{2} / b\right)$. 
Table 3 Summary of properties and results per spreadsheet category

\begin{tabular}{|c|c|c|c|c|c|c|}
\hline & \multicolumn{2}{|c|}{ Exercises (9) } & \multicolumn{2}{|c|}{ Tutorials (21) } & \multicolumn{2}{|l|}{ Data (4) } \\
\hline & Overall & Sheet avg. & Overall & Sheet avg. & Overall & Sheet avg. \\
\hline Tables & 19 & 2.11 & 48 & 2.29 & 4 & 1 \\
\hline Cells & 1231 & 137 & 1889 & 90 & 2320 & 580 \\
\hline Intended constraints & 34 & 3.78 & 52 & 2.48 & 6 & 1.50 \\
\hline Recall (all) & 0.88 & 0.85 & 0.88 & 0.87 & 1.00 & 1.00 \\
\hline Recall (supported) & 1.00 & 1.00 & 1.00 & 1.00 & 1.00 & 1.00 \\
\hline Precision & 0.94 & 0.96 & 0.69 & 0.90 & 1.00 & 1.00 \\
\hline Runtime TaCLe & 0.91 & 0.10 & 0.87 & 0.04 & 0.79 & 0.20 \\
\hline Runtime no-CSP baseline & 58.36 & 6.48 & 36.84 & 1.75 & 19.73 & 4.93 \\
\hline
\end{tabular}

\subsection{Effectiveness on real spreadsheets}

In order to test our approach on real data we assembled a benchmark of spreadsheets from three sources: (1) spreadsheets from an exercise session for teaching Excel at the Brussels Business School based on the book (Van den Broeck and Cuypers 2011), covering the most popular Excel formulas; (2) spreadsheets from online tutorials on Excel formulas; and (3) publicly available data spreadsheets such as real-world crime statistics (FBI:UCR) or financial reports (US BEA). The data and its extended description are available in "Appendix 1 " in Table 4 and at the same repository as before with the links to all original publicly available spreadsheets used in the experiments.

Table 3 gives an overview of the spreadsheets in the different categories and summarizes the results of our experiments. The data is also visualized in Fig. 4.

$Q_{1}$ : Effectiveness Our first question, $Q_{1}$, is about the inability to find intended constraints due to excessive runtime. As Table 3 (last row) shows, the runtime of our method on these spreadsheets is always fast. The average runtime across all spreadsheets is $0.08 s$. Across categories the average running times fluctuate in the range between $0.04 s$ to $0.20 s$. While most spreadsheets can be processed very quickly, running times can go up to around $0.61 s$ for others. In the previous section we analyzed the effect of various factors that can help explain runtime behavior on data generated to allow many candidate subassignments (e.g. using only vectors of the same type and length). Our benchmark experiments show that our algorithm is able to perform well on real spreadsheets, even for larger numbers of vectors. Moreover, these experiments validate our two-step approach, since TaCLe is able to find constraints 20 to 60 times faster than the no-CSP baseline.

Looking at the runtime per constraint template individually, the slowest by far is fuzzy lookup using $\pm 21 \%$ of the running time. It is followed by product $( \pm 7 \%)$, relative difference $( \pm 6 \%)$, running total $( \pm 5 \%)$, difference $( \pm 5 \%)$ and various row-aggregates $( \pm 5 \%)$.

Dependencies are optional and do not affect precision or recall. However, our method can exploit dependencies in order to find constraints incrementally and increase the systems efficiency. To illustrate the effect this can have, we ran the benchmarks without using $A S C E N D I N G$ as base constraint for fuzzy lookup. The total running times per category increase by $74 \%$ (exercises), $84 \%$ (tutorials) and $13 \%$ (data). This shows that the use of dependencies can have a strong effect on running times by reducing the number of candidate assignments for depending constraints and sharing the computation and pruning done for one constraint with another. 


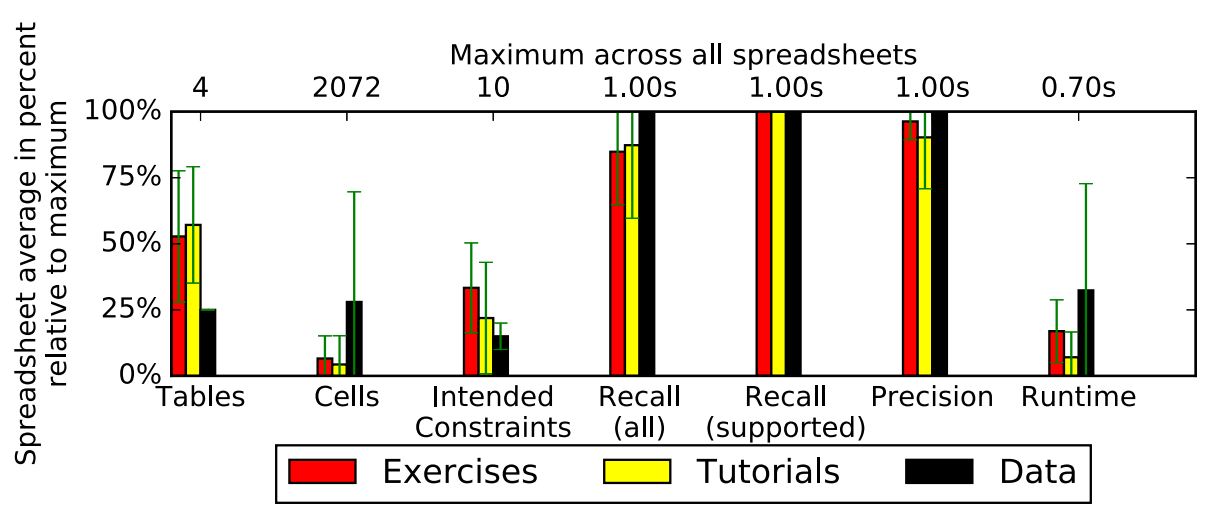

Fig. 4 This figure visualizes the data from Table 3, i.e., the average properties and performance of spreadsheets within every benchmark category. Values are scaled relatively to the maximum value across all individual spreadsheets. Green bars indicate standard deviation within the category (Color figure online)

$Q_{2}$ : Intended and supported constraints The overall recall achieved by our system, TaCLe, is 0.88 (81/92). When looking at the recall per category, TaCLe is able to consistently obtain high values for both overall and average recall, as shown in Table 3. The intended constraints not discovered correspond to constraints that are not supported by the system, hence the supported recall is 1 . Therefore, TaCLe is able to find all constraints for supported constraint templates, which shows that our method is able to learn all constraints using the bias chosen, that is, the formalism of signature and definition.

The intended constraints that are not discovered correspond with nested constraints (e.g., $R=\frac{W}{(L / 100)^{2}}$ ), variants of supported constraints (e.g., series that do not start at 1) or more complex versions of supported constraints (e.g. conditional aggregates whose conditions are constant values).

$Q_{3}$ : Precision The final question, $Q_{3}$, is about the amount and type of non-intended constraints found. Across all spreadsheets TaCLe achieves a precision of $0.79(81 / 103)$. While the average precision per spreadsheet is high, over 0.90 in every category, we observe that the overall precision is much higher for the exercises and data categories (0.94 and 1.00) than for the tutorials category (0.69).

Examining the tutorial spreadsheets confirms that there are a few spreadsheets that have a high number of additional constraints, e.g., one spreadsheet computes aggregates on inventory data, but copies the data column for every aggregate. Since every aggregate can be calculated based on any of the equal columns, our method will find all of these constraints. However, for every aggregate only one constraint is considered to be intended, the others will be considered as redundant and decrease the precision.

Such redundant or unintended constraints are the result of multiple ways to calculate the same result, e.g., if there is duplicate data as in the example above. One way to increase precision for this case is to post-process the constraints to detect equivalences and implications, and heuristically select one among multiple ways to compute the same vector.

\section{Applications}

In this section, we illustrate how two of the motivating applications, auto-completion and error checking, can build on our method for solving the tabular constraint learning problem. 


\begin{tabular}{|l|rrr|}
\hline \multicolumn{1}{|c}{ Date } & Units & \multicolumn{2}{c|}{ Total Amt. } \\
\hline $24 / 06 / 2012$ North & 186 & $\$ 50,592.00$ \\
$01 / 06 / 2012$ East & 356 & $\$ 96,832.00$ \\
$09 / 09 / 2012$ West & 907 & $\$ 246,704.00$ \\
$26 / 06 / 2012$ South & 190 & $\$ 51,680.00$ \\
$22 / 04 / 2012$ North & 717 & $\$ 195,024.00$ \\
$22 / 03 / 2012$ West & 550 & $\$ 149,600.00$ \\
$19 / 12 / 2011$ East & 942 & $\$ 256,224.00$ \\
$31 / 10 / 2011$ North & 901 & $\$ 245,072.00$ \\
$02 / 10 / 2011$ West & 117 & $\$ 31,824.00$ \\
\hline \multicolumn{2}{|c|}{ B1 = T1[: 1:2] } & \multicolumn{3}{|c|}{ B2 $=$ T1[: 3:4] } \\
\hline
\end{tabular}

$\begin{aligned} & \text { Region } \\
& \text { B3 = T2[:, 1] }\end{aligned}$
\begin{tabular}{|l|rr|}
\hline North & 1804 & 490688 \\
East & 1298 & 353056 \\
South & 190 & 51680 \\
\hline West & 1574 & 428128 \\
\hline New data (DD) & Suggested & Suggested \\
\hline
\end{tabular}

Fig. 5 Auto-completion works by learning constraint on a snapshot (green tables $T_{1}$ and $T_{2}$ ) and combining those constraints with new data $\Delta D$ (purple). For example, in this figure a user types a new value West. The system can then suggest values (blue) for the other cells in that row (Color figure online)

\subsection{Auto-completion}

Auto-completion can be seen as using knowledge derived from the current data to predict new values before they are written by the user. We can decompose this process into three phases: (1) learn constraints on a snapshot of the spreadsheet; (2) new data $(\Delta D)$ is added by the user; and (3) the system uses the learned constraints to predict missing values.

Step 1 corresponds to running TaCLe on the snapshot to discover constraints in the spreadsheet. In step 2, the user extends one or more vectors in the spreadsheet with new values. These extended vectors now stretch beyond the original table they are situated in. Therefore, the remaining vectors and blocks in the same table are marked as incomplete, indicating they are missing some values required to complete the table. Finally, in step 3, functional constraints discovered in step 1, are used to predict missing values. A functional constraint, such as $B_{r}=S U M_{c o l}\left(\mathbf{B}_{\mathbf{x}}\right)$, calculates a result $\left(B_{r}\right)$ based on its input $\left(\mathbf{B}_{\mathbf{x}}\right)$. Hence, a functional constraint can predict missing values if the result block is incomplete and none of the input blocks is incomplete.

Let us illustrate this application on the two-table spreadsheet shown in Fig. 5. First, we run TaCLe on tables $T_{1}$ and $T_{2}$ (in green) and discover the constraints $T_{2}[:, 2]=\operatorname{SUMIF}\left(T_{1}[\right.$ : , 2], $\left.T_{2}[:, 1], T_{1}[:, 3]\right)$ and $T_{2}[:, 3]=\operatorname{SUMIF}\left(T_{1}[:, 2], T_{2}[:, 1], T_{1}[:, 4]\right)$. Afterwards, vector $T_{2}[:, 1]$ is extended with a new value West and vectors $T_{2}[:, 2]$ and $T_{2}[:, 3]$ are marked as incomplete. Finally, the SUMIF constraints are used to complete their incomplete result blocks, suggesting $T_{2}[4,2]=1547$ and $T_{2}[4,3]=428128$.

In an interactive setting, auto-completion can be performed whenever new values are entered, however, to update the learned constraints, TaCLe would need to consider only assignments containing modified vectors.

\subsection{Error detection}

We discuss two cases of error detection. The first setting is the online detection of errors and is similar to the auto-completion setting. Constraints are learned on a snapshot before new data is added. Functional constraints whose result block was modified are checked on the new data. If such a constraint does not hold anymore, it could indicate errors such as typing errors in the data just entered. For example, filling in $T_{2}[4,2]=1572$ in Fig. 5 would violate the corresponding SUMIF constraint and likely indicate an error.

The second setting is offline error detection, that is, on a given file. First, TaCLe is run to detect all constraints $(S)$ in the given spreadsheet. Then, from this spreadsheet, one can repeatedly remove one or multiple rows or columns $(v)$ and detect all constraints $\left(S_{v}\right)$ that hold in the modified spreadsheet. Every constraint in $S_{v}$ that did not occur in $S$ is violated 
by a value in the rows or columns $v$ that were removed. Therefore, values in the rows or columns $v$ that occur in such a violated constraint can be marked as potential errors to be reviewed by a user. This approach allows constraint discovery to be treated as a black-box, however, it requires a scheme for choosing which row(s) or column(s) are removed.

Alternatively, we propose to extend current constraint definitions to tolerate up to a given number of wrong values and report these. This approach would be more robust, however, it requires definition tests to be able to deal with wrong values.

\section{Conclusions}

Our goal is to automatically identify constraints in a spreadsheet. We have presented and evaluated our approach, implemented as the system TaCLe, that is able to learn many different constraints in tabular data. The resulting method has high recall, produces a limited number of redundant constraints and is sufficiently efficient for normal and interactive use. Our approach also allows new constraint templates to be easily added by specifying their signature in terms of properties and providing an implementation that finds subassignments satisfying the definition.

The approach is designed to find constraints that hold over entire columns and rows. In future work we plan to extend this to learn arbitrary nested constraints (e.g. $B_{4}=\left(B_{1}+\right.$ $\left.B_{2}\right) / B_{3}$ ), as well as constraints over only a subset of the vectors. This may give rise to more redundant and spurious constraints, which was not problematic up to this point.

Two promising ways to mitigate redundant and spurious constraints are heuristic filtering or post-processing of the constraint set and the integration of our approach in an interactive setting where users can receive and provide feedback. The latter is more similar to an active learning setting.

A final direction is that the current approach assumes consistent (noise-free) data, typically generated by some external system. We do support the detection of limited precision calculations as this is a type of noise generated by exporting data. Also, our approach can be used for error correction in case some noise/errors are assumed. However, in inductive logic programming there has been much work on how to handle constraints over noisy data such as allowing a tolerance level $\epsilon$ when a rule or a constraint $\sum_{i=1}^{n} b_{i}=c$ matches up to $\epsilon$ : $\left|\sum_{i=1}^{n} b_{i}-c\right| \leq \epsilon$, while in constraint satisfaction a popular alternative is to consider soft constraints that can be violated at a cost, which is a real value, and then the task is to find the top- $k$ best matching constraints. Threshold bounded error tolerance can be supported by adapting constraint definitions to allow such noise. This could extend the approach to new application domains, beyond traditional spreadsheets.

Acknowledgements This work has been partially funded by the ERC AdG SYNTH (Synthesising inductive data models) and a PhD and Postdoctoral Fellowship of the Research Foundation-Flanders.

\section{Appendix 1: Spreadsheet dataset overview}

We collected spreadsheets from three main sources.

- After identifying popular Excel functions, the MS Office web page has an overview of popular functions (see the link in Sect. 4.4.3), we searched for online tutorials about these functions and collected them under the category Tutorials. A link to each webpage found and used is provided in the accompanying GitHub repository. 
Table 4 Constraint occurrence in the collected dataset in absolute values by category

\begin{tabular}{lllc}
\hline Constraint & Exercises & Data & Tutorials \\
\hline Average (col) & 0 & 0 & 2 \\
Average (row) & 0 & 1 & 0 \\
Average-if & 1 & 0 & 0 \\
Count (col) & 0 & 0 & 1 \\
Count-if & 1 & 0 & 0 \\
Difference & 2 & 1 & 0 \\
Equal & 0 & 0 & 10 \\
Foreign-product & 1 & 0 & 0 \\
Fuzzy-lookup & 2 & 0 & 1 \\
Lookup & 1 & 0 & 0 \\
Max (col) & 0 & 0 & 2 \\
Max-if & 1 & 0 & 0 \\
Min (col) & 0 & 0 & 3 \\
min-if & 1 & 0 & 0 \\
Percentual-diff & 5 & 1 & 0 \\
Product & 2 & 0 & 3 \\
Project & 1 & 0 & 0 \\
Rank & 1 & 0 & 0 \\
Series & 2 & 1 & 0 \\
Sum (col) & 5 & 0 & 10 \\
Sum (row) & 2 & 2 & 3 \\
Sum-if & 2 & 0 & 9 \\
Sum-product & 0 & & 2 \\
\hline & & 0 &
\end{tabular}

- We have collected the exercises under the category Exercises from the introductory Excel book (Van den Broeck and Cuypers 2011) that focused 1) on popular Excel functions and 2) on datatypes supported by our system.

- We have collected under the category Data spreadsheets reporting data. More specifically, economic data, crime reporting data and data from runtime experiments. The spreadsheets on economic data and crime reporting are publicly available and originate from the U.S. Bureau of Economic Analysis (BEA), the RWE annual report 2014 and the U.S. FBI Uniform Crime Reporting (UCR) Program. The links are also provided in the accompanying GitHub repository) and data summary spreadsheets.

The overview of constraint distributions per category is presented in Table 4.

The file links.txt, available in the accompanying GitHub repository, contains the links to all original publicly available spreadsheets used in the experiments.

\section{Appendix 2: Examples of learned constraints}

This section provides examples of actual constraints learned by TaCLe on real spreadsheets from our benchmark dataset. Figure 6 shows constraints learned on a dataset containing conditional aggregates. In Fig. 7 a dataset is used that contains arithmetic constraints. More examples and screenshots can be found in the accompanying GitHub repository: https:// github.com/SergeyParamonov/TaCLe. 


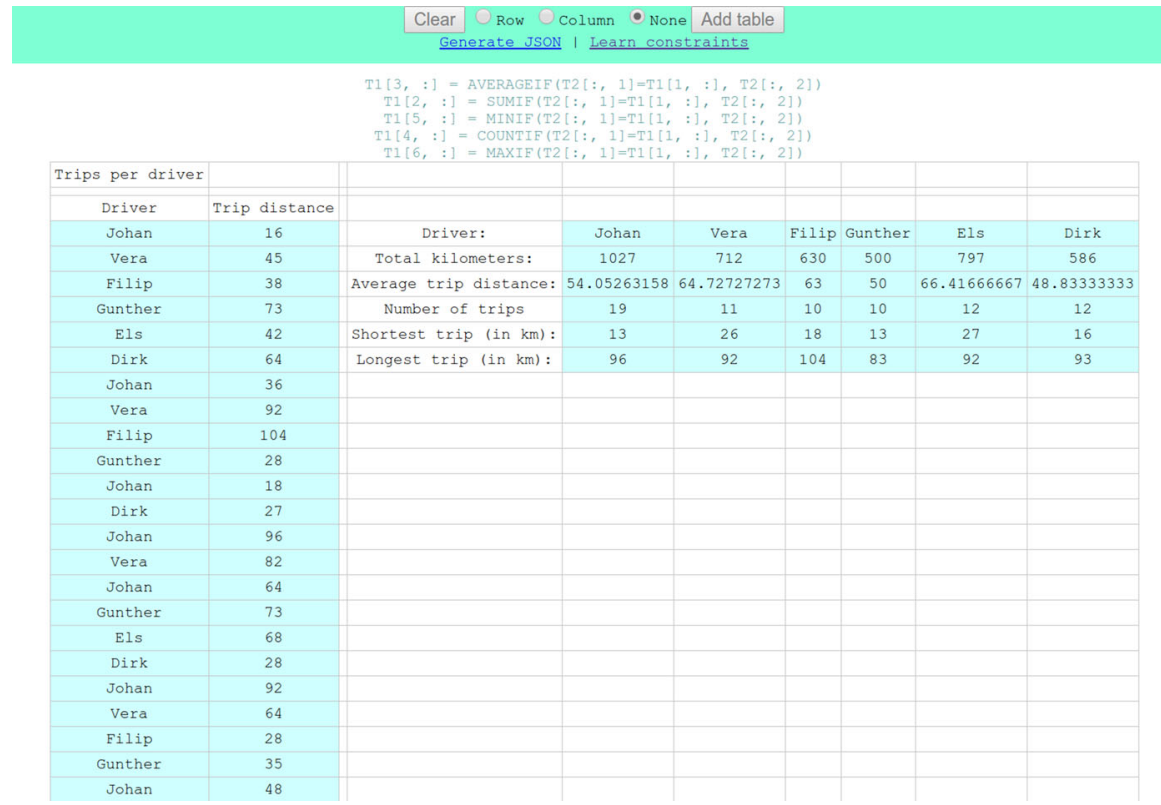

Fig. 6 This screenshots shows the five constraints learned from a spreadsheet containing conditional aggregates. The first table extends below the depicted area. (Translated spreadsheet from exercises (Van den Broeck and Cuypers 2011), originally in Dutch.)

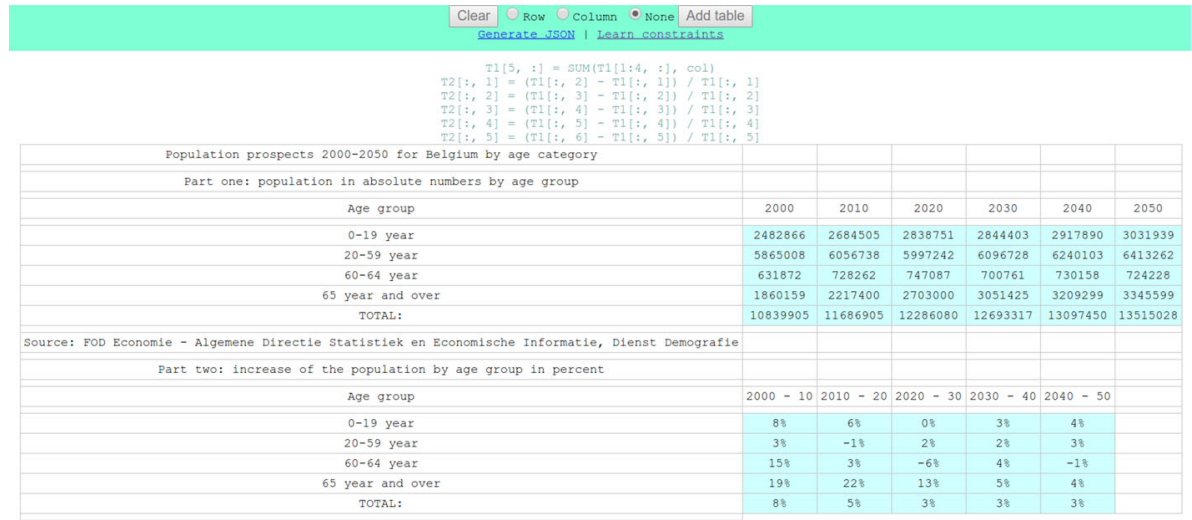

Choose File belgium_english.csV

Fig. 7 This screenshot shows the six constraints learned on a spreadsheet containing arithmetic constraints: sum and relative difference. (Translated spreadsheet from exercises (Van den Broeck and Cuypers 2011), originally in Dutch.)

\section{References}

Beldiceanu, N., \& Simonis, H. (2012). Principles and practice of constraint programming. In M. Milano (Ed.), A model seeker: Extracting global constraint models from positive examples (pp. 141-157). Berlin: Springer.

Bessiere, C., Coletta, R., Hebrard, E., Katsirelos, G., Lazaar, N., Narodytska, N., Quimper, C., \& Walsh, T. (2013). Constraint acquisition via partial queries. In IJCAI. 
Bessière, C., Coletta, R., Koriche, F., \& O’Sullivan, B. (2005). A SAT-based version space algorithm for acquiring constraint satisfaction problems. In Proc. of ECML.

De Raedt, L. (2008). Logical and Relational Learning. New York: Springer.

De Raedt, L., \& Dehaspe, L. (1997). Clausal discovery. Machine Learning, 26(2-3), 99-146.

Fang, J., Mitra, P., Tang, Z., \& Giles, C. L. (2012). Table header detection and classification. In AAAI.

Flach, P. A., \& Savnik, I. (1999). Database dependency discovery: A machine learning approach. AI Communications, 12(3), 139-160.

Gordon, A. D., Graepel, T., Rolland, N., Russo, C., Borgstrom, J., \& Guiver, J. (2013). Tabular: A schemadriven probabilistic programming language. Technical report MSR-TR-2013-118, December 2013.

Gulwani, S. (2011). Automating string processing in spreadsheets using input-output examples. In $A C M$ SIGPLAN-SIGACT symposium on principles of programming languages, POPL, pp. 317-330, New York, NY, USA, 2011. ACM.

Herndon, T., Ash, M., \& Pollin, R. (2013). Does high public debt consistently stifle economic growth? A critique of Reinhart and Rogoff. Cambridge Journal of Economics, 38(2), 257-279.

Huhtala, Y., Kärkkäinen, J., Porkka, P., \& Toivonen, H. (1999). Tane: An efficient algorithm for discovering functional and approximate dependencies. Computer Journal, 42, 100-111.

Isakowitz, T., Schocken, S., \& Lucas, H. C, Jr. (1995). Toward a logical/physical theory of spreadsheet modeling. ACM Transactions Information Systems, 13(1), 1-37.

Lallouet, A., Lopez, M., Martin, L., \& Vrain, C. (2010). On learning constraint problems. ICTAI, 1, 45-52.

Le, V., \& Gulwani, S. (2014). Flashextract: A framework for data extraction by examples (p. 55). In ACM SIGPLAN conference on programming language design and implementation: PLDI.

Mannila, H., \& Räihä, K.-J. (1994). Algorithms for inferring functional dependencies from relations. DKE, 12(1), 83-99.

Mansinghka, V. K., Tibbetts, R., Baxter, J., Shafto, P., Eaves, B. (2015). Bayesdb: A probabilistic programming system for querying the probable implications of data. arXiv:1512.05006v1

Niemeyer, G. (2017). https://labix.org/python-constraint.

Reinhart, C. M., \& Rogoff, K. S. (2010). Growth in a time of debt. Working paper 15639, National Bureau of Economic Research, January 2010.

Savnik, I., \& Flach, P. A. (2000). Discovery of multivalued dependencies from relations. Intelligent Data Analysis, 4(3-4), 195-211.

Todorovski, L. (2010). Equation discovery. In C. Sammut \& G. I. Webb (Eds.), Encyclopedia of machine learning (pp. 327-330). Boston, MA: Springer.

Van den Broeck, E., Cuypers, E. (2011). MS Excel 2010. Uitgeverij De Boeck Hoger en universitair onderwijs. ISBN: $9045534436,9789045534435$. 